\title{
El camino de la kata en \\ el judo Kodokan
}

Llyr C. Jones \& Michael J. Hanon

Investigadores independientes (Reino Unido)

Es traducción del artículo The Way of Kata in Kodokan Judo, publicado en el Journal of Asian Martial Arts, volumen 19, número 4 (8-37), 2010

\section{Resumen}

Este artículo presenta una "inmersión profunda" en los ejercicios formales (katas) del judo Kodokan. De forma específica, tiene dos objetivos: (i) explicar el propósito de la kata en judo, y (ii) evaluar de manera crítica el concepto de campeonatos de katas. Para alcanzar estos objetivos, ofrecemos los comentarios personales de los autores, observaciones de fuentes japonesas poco frecuentes, así como muestras del pensamiento de expertos mundiales de judo. Concluimos que el concepto de campeonatos de katas es fundamentalmente erróneo y que además daña el entrenamiento de las katas.

Palabras clave: Jigoro Kano, formas, entrenamiento, competición, desarrollo personal.

\section{The Way of Kata in Kodokan Judo}

Abstract: This article presents a "deep dive" into the formal exercises (katas) of Kodokan judo. Specifically, it has two objectives: (i) to explain the purpose of kata in judo, and (ii) to critically evaluate the concept of kata championships. To achieve these objectives, we offer the authors' personal comments, observations from rare Japanese source material, as well as insight into the thinking of world-renowned judo experts. We conclude that the concept of kata championships is fundamentally flawed and also damaging kata training.

Key words: Jigoro Kano, forms, training, competition, personal development.

\section{O caminho do kata no judo Kodokan}

Resumo: Este artigo apresenta uma "imersão profunda" nos exercícios formais (katas) do judo Kodokan. De forma específica, tem dois objectivos: (i) explicar o propósito do kata no judo, e (ii) avaliar criticamente o conceito dos campeonatos de katas. Para alcançar esses objectivos, procuramos referir os comentários pessoais dos autores, observações pouco frequentes de fontes japonesas, assim como revelar o pensamento de especialistas mundiais de judo. Concluímos que o conceito de campeonatos de kata é fundamentalmente erróneo e que estraga 0 treino dos katas.

Palavras-chave: Jigoro Kano, treino, competição, desenvolvimento pessoal. 


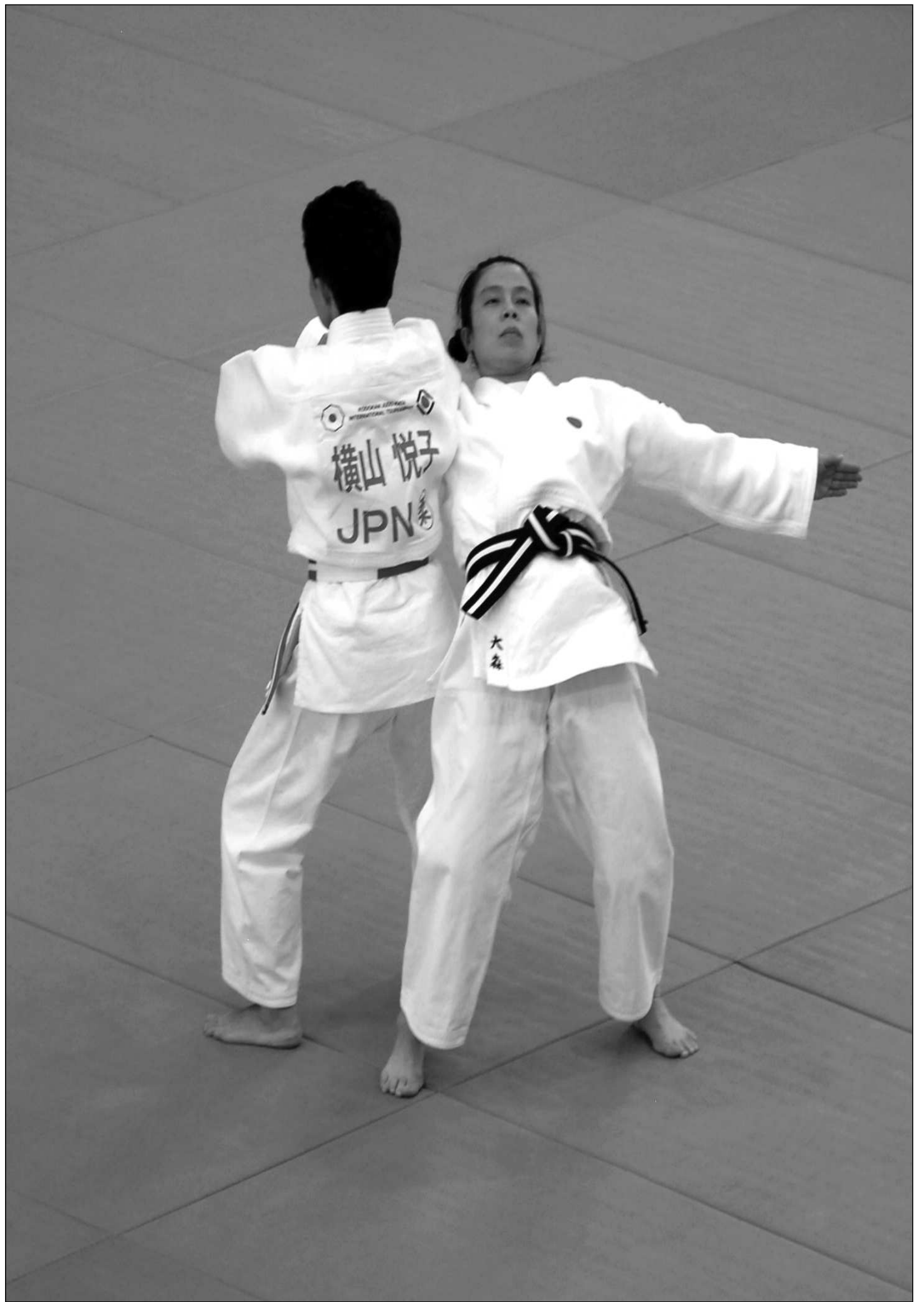

8

Jones $\&$ Hanon $\diamond$ El camino de la kata en el judo Kodokan Revista de Artes Marciales Asiáticas $\diamond$ Volumen 6 Número 1 (7-34) $\diamond 2011$ 


\section{El camino de la kata en el judo Kodokan}

Llyr C. Jones \& Michael J. Hanon

Investigadores independientes (Reino Unido)

\section{Introducción}

En un artículo, De Crée y Jones (2009) explican cómo el Judo Kodokan'1, desarrollado por Kano Jigoro (1860-1938), constituye una educación cooperativa completa, un sistema de enseñanza (pedagogía) y una filosofía -basada, entre otras cosas, en valores neo-confucianos, escuelas de artes marciales japonesas tradicionales y modernos principios educativos occidentales-. En particular, el judo enfatiza el valor educativo holístico del entrenamiento en el ataque y la defensa, de modo que puede ser un "camino", o forma de vida, del cual puede participar y beneficiarse todo el mundo. De este modo, y contrariamente al debate popular, el judo no es ni un "arte marcial" ni un "deporte de combate".

[] Judo en el sentido amplio trata de trascender el contexto deportivo y... Incluye aspectos relacionados con la educación, la salud, así como con la defensa personal, tanto física como emocional.

\section{- Gutiérrez García, Pérez Gutiérrez \& Svinth (2010:127)}

La más completa definición de judo disponible en la literatura la brinda 0 da Join² (1929). En esta obra, utiliza y se fundamenta en una cita original de Kano Jigoro que apareció en la revista Judo editada por el Kodokan (Kano, 1915). O da escribe: "Judo es el modo más efectivo de utilizar el poder de la mente y el cuerpo. Su entrenamiento cultiva el cuerpo y el espíritu a través de la práctica del ataque y la defensa; la esencia de ese código moral (o "camino") de principios es el aprendizaje a través de la autoconsciencia. Por tanto, el judo fue innovado teniendo en cuenta que el objetivo último fuese la perfección de uno mismo y el beneficio obtenido de la vida. En resumen, el judo es el modo más efectivo de usar la mente y el cuerpo para el beneficio propio y el de los demás" (O da, 1929:8).

Kano Jigoro expresó los conceptos nucleares del judo a través de los dichos "buen uso de la mente y el cuerpo" y "bienestar y beneficio mutuos". Un espíritu de generosidad y asistencia mutua es por tanto integral en el judo. Kano concibió que, a través de la aplicación de los principios del judo a la vida cotidiana, un practicante de judo alcanza equilibrio y dominio de sí mismo, llegando a estar

1 El Kodokan, centro principal del judo, fue fundado en 1882 por Kano Jigoro, el creador del judo.

2 Muchas publicaciones escriben erróneamente Tsunetane como primer nombre de 0 da, en lugar de Join. 
mejor preparado para lidiar con el estrés de las rutinas e interactuar con otros seres humanos de formas positivas y mutuamente beneficiosas.

\section{Sobre las técnicas de judo}

Los aspectos físicos de las técnicas de judo se basan principalmente en los estilos de las escuelas tradicionales de jujutsu Tenjin Shin'yo-ryu, Kito-ryu y Yoshin-ryu. Sin embargo, como ya se ha comentado, el aspecto más significativo del judo es su combinación de educación física y mental para ayudar a sus practicantes a desarrollar cuerpos bien equilibrados y mentes que, mediante un espíritu de generosidad y asistencia mutua, actúen para la mejora de la sociedad en su conjunto. Como tales, las técnicas de judo son simples vehículos hacia metas mayores. Ellas, en sí mismas, no son el objetivo. Las técnicas de judo tienen utilidad sólo como herramientas que permiten al practicante de judo a darse cuenta de esas aspiraciones más altas.

\section{Los elementos de instrucción en judo}

Escribiendo en la revista Kokushi a los pocos años del nacimiento del Kodokan, Kano (1899) explica cómo la instrucción del judo consta de cuatro componentes:

- Práctica libre (randori): sesiones de práctica con adversario en las cuales ambos participantes desarrollan el ataque y la defensa, utilizando de forma libre técnicas de proyección y/o inmovilización (Kawamura \& Daigo, 2000:109-110).

- Formas (katas): ejercicios sobre patrones de movimientos formales que contienen los modelos ideales de los movimientos, ilustrando principios de combate específicos (Ibid: 86).

- Lecciones (kogi): las lecciones sobre los principios del judo se consideraban parte esencial de la educación en judo. Kano creía que el entrenamiento para las competiciones o el combate se veía ampliamente mejorado mediante la enseñanza teórica a través de lecciones.

- Diálogo (mondo): las sesiones de "pregunta y respuesta" entre los alumnos y el profesor también eran consideradas importantes para reforzar el aprendizaje práctico y formal.

Murata Naoki, el actual conservador del museo y biblioteca del Kodokan, añade la competición (shiai) a esa lista, afirmando que los torneos son un importante componente suplementario en el proceso de aprendizaje del judo (Murata, 2007).

También es importante reconocer que el modo de enseñanza del judo expuesto anteriormente nunca se puso en práctica fuera de Japón. No obstante, una excepción destacable la encontramos en las clases que Trevor Leggett (19142000) impartía los domingos (con asistencia sólo por invitación) en el Budokwai de Londres. Hoare (2000) describe cómo esas sesiones "estaban siempre llenas", siendo la clase "una mezcla de trabajo extenuante, competición e instrucción sobre todos los aspectos del judo". De modo similar, Hicks (1996) recuerda que para Leggett "el judo era un entrenamiento ético y educativo que abría las puertas a la 
comprensión más allá del dojo" y cómo incluso "sus alumnos más entregados se alarmaban a veces cuando se les pedía que escribieran ensayos".

¿Por qué nunca se estableció y cuajó una tradición de educación formal en judo a través de las lecciones y el diálogo fuera de Japón? En los años formativos del judo era el mismo Kano y otros profesores mayores los que dirigían esas lecciones y diálogos. A pesar de que Kano y otros mayores realizaron una cantidad considerable de viajes internacionales, mantuvieron en Japón su residencia habitual. A excepción de algunos casos contados, no existían en otros lugares profesores de conocimiento comparable que pudiesen dirigir el aprendizaje formal. Por esta razón no llegó a establecerse tal práctica de instrucción.

Más aún, con la continua internacionalización del judo y el énfasis creciente de la Federación Internacional de Judo (IJF) hacia el deporte competitivo, incluso la práctica de la kata se marginó y fue vista como una rareza histórica. Todo esto dejó tras de sí un tipo de judo menor y distorsionado que consistía sólo en práctica libre y competición, que es lo que la mayoría de los judokas actuales piensan que compone la totalidad del judo.

\section{La práctica de las katas en la actualidad}

En el artículo "Competition, Kata and the Art of Judo" ["Competición, Kata y el arte del judo"] (Jones, 2005) se explicaba cómo la práctica de katas en judo se había atenuado. Señalaba que el énfasis de la IJF y los organismos de gobierno nacionales (NGB) en los aspectos deportivo-competitivos del judo y en ganar medallas habían producido generaciones de luchadores y entrenadores que consideraban la práctica de la kata como algo anticuado y sin ningún valor o relevancia.

Este enfoque fue más tarde respaldado por Alessio Oltremari en un artículo en la red: "La competición y el combate ha encandilado a generaciones de practicantes de judo y al aplicar principios de muchos deportes al judo, han olvidado sus orígenes y su significado. Lo importante es ganar -no importa si la técnica utilizada es sucia, vulgar y peligrosa -. Que la técnica esté ausente no importa si la fuerza bruta es suficiente para ganar. Mejor estar haciendo entrenamiento con pesas que gastar el tiempo profundizando en la técnica" (Oltremari, s.f.).

Sin embargo, recientemente se está produciendo un resurgir en la popularidad de la kata, pero no dirigido por la apreciación de la mejora personal que se obtiene de su práctica regular, sino por las oportunidades de ganar medalla que provee el fenómeno de rápida expansión de los "campeonatos de katas". De hecho, tal es la popularidad de las katas como logro competitivo que el desarrollo de estrategias de entrenamiento para tener éxito en los campeonatos está empezando a considerarse como un asunto de investigación (por ejemplo, véase Sheedy, 2010).

\section{Las katas del judo Kodokan}

\section{La kata como libro de texto viviente}

La práctica de las katas es un importante método de entrenamiento en la mayoría de las artes marciales japonesas tradicionales y modernas. Finn (1991:211) 
se muestra especialmente perspicaz cuando escribe que las katas son "formas preestablecidas ${ }^{3}$ en las artes marciales japonesas que son como libros de texto vivientes. Contienen toda la información fundamental en una forma animada, con la cual perfeccionar la técnica y la comprensión de la habilidad particular".

\section{Las katas catalogadas}

Existen diez katas "catalogadas" 4 en el Kodokan (de Crée \& Jones, 2009). Eso a pesar de que varias fuentes con cierta popularidad afirmen de forma incorrecta que sólo hay siete, ocho o nueve katas oficiales del Kodokan. Las diez katas están detalladas en la Tabla 1, la cual se estructura según cómo las katas son normalmente categorizadas según su propósito (Kotani, Osawa \& Hirose, 1968:1; Otaki \& Draeger, 1983:32-33).

\section{Tabla 1: las katas del Judo Kodokan}

FORMAS DE PRÁCTICA LIBRE

- Formas de proyección.

- Formas de agarrar o coger.

FORMAS DE DEFENSA PERSONAL

- Formas de decisión.

- Formas de defensa personal del Kodokan.

- Métodos de defensa personal para mujeres.

FORMAS DE EDUCACIÓN FÍSICA

- Formas de suavidad \& flexibilidad

- Formas del (uso apropiado) de la fuerza

- Educación física nacional de acuerdo al (principio de) mejor uso de la energía

\section{FORMAS DE TEORÍA}

- Las cinco formas

- Las formas antiguas
RANDORI-NO-KATA

- Kage-no-kata

- Katame-no-kata

SHOBU-NO-KATA

- Kime-no-kata

- Kodokan Goshin Jutsu

- Joshi Goshin Ho

RENTAI-N O-KATA

- Ju-no-kata

- Go-no-kata

- Sei-ryoku-zen'yo Kokumin-Taiiku

RI-NO-KATA

- Itsutsu-no-kata

- Koshiki-no-kata

3 Uno de los problemas al traducir palabras de un lenguaje a otro es que existen sutilezas y pequeños matices de la palabra original que se pierden. Esto ocurre en gran medida con la palabra kata. Aunque la traducción aceptada de kata en judo es "forma", otras palabras, tales como "plantilla", "patrón" o "estilo", serían más adecuadas. La palabra kata se utiliza a menudo en la cultura japonesa para describir estándares de posturas. Esto también sería apropiado.

4 El término "kata catalogada" se debe a John Cornish (70 Dan de judo y 80 Dan de aikido) (Cornish, 2004). Se refiere al grupo formalmente escrito de ejercicios coreografiados y técnicas que ilustran principios específicos en contraposición a secuencias de técnicas de judo preestablecidas ad hoc. 
En su lección inaugural del 2008 Kodokan Summer Kata Course [Curso de verano de katas Kodokan 2008], Daigo Toshiro 5 ofreció una breve panorámica general con algo de historia de cada una de las katas reconocidas y practicadas en la actualidad, enumerando nueve katas distintas en total (Kano, s.f., en Daigo, 2008; Kotani \& Otaki, s.f, en Daigo, 2008-2009). La lección de Daigo subsiguientemente formó la base para su amplio artículo sobre katas, publicado en siete partes en una serie aparecida en la revista del Kodokan, Judo (Daigo, 2008-2009).

Contando nueve katas del Kodokan, Daigo, de forma inexplicable, omite la kata de defensa personal femenina, Joshi Goshin Ho, que está oficialmente aprobada si bien es una kata poco común del Kodokan. Además, el recuento de las katas del Kodokan ignora tanto las "Formas de decisión" (Kime Shiki) como las "Formas de la suavidad" (Ju Shiki), dos katas que se consideraban separadas en la antigüedad, pero que hoy en día son consideradas como parte de Seiryoku-zen'yo Kokumin-Taiiku. Existen además numerosas katas no aprobadas por el Kodokan, de origen tanto japonés como extranjero. Este artículo no considerará ninguna de las katas no aprobadas.

Está más allá del objetivo del presente artículo el presentar los detalles técnicos ("el cómo") de las diez katas del Kodokan. Los detalles técnicos completos de ocho de las diez katas (siendo omitidas la Joshi Goshin Ho y la Go-no-kata) se ofrecen en el libro Judo Kodokan ${ }^{6}$ (Kano, 1986: 145-251). Para los detalles técnicos de la Joshi Goshin Ho, dirigimos al lector al texto Kata of Kodokan Judo (Kotani et al, 1968: 124-153). De manera similar, para los detalles técnicos de la Go-no-kata, el lector puede remitirse al reciente cuadernillo de Mori (2008).

\section{El propósito de la práctica de katas en judo}

En los primeros tiempos del Kodokan, el método de enseñanza de Kano Jigoro consistía en iniciar al estudiante en la práctica libre y dejarle asimilar las katas de forma natural. Sin embargo, este sistema se convirtió pronto en algo insostenible.

Escribiendo en el periódico del Kodokan Sakko ("Despertar") en 1927, Kano Jigoro explicaba cómo las katas fueron creadas como un marco de enseñanza para dar respuesta al hecho de que el Kodokan se estaba expandiendo rápidamente y que él (Kano) no podía enseñar su programa únicamente él mismo y no había instructores entrenados. Kano escribió: "La razón principal de por qué las katas fueron creadas fue por el cada vez mayor número de alumnos en el Kodokan. Enseñar a los estudiantes de forma individual se convirtió en algo imposible, tal y como sucedía en los primeros tiempos, y se requería un sistema de enseñanza para muchos estudiantes a la vez" (Kano, 1927).

Existe una creencia bastante extendida, pero errónea, acerca de que el objetivo de la práctica de las katas en judo es el desarrollo del refinamiento técnico y

5 Daigo Toshiro (n. 1926) fue durante muchos años el instructor jefe del Instituto del Judo Kodokan. Fue promocionado a 10 Dan en 2006 y es posiblemente el mayor experto mundial en katas. Véase Sidney (2003:144-153) para un detallado perfil de Daigo.

6 Aunque Kano Jigoro se presenta como autor de este libro, es de hecho una compilación del Instituto Kodokan que data de una fecha muy posterior a la muerte del fundador. 
la perfección de la técnica?. Este es tan solo un componente menor de la práctica de las katas, ya que la técnica puede ser perfeccionada de otro modo. El estudio apropiado de las katas contribuye a mejorar la comprensión ${ }^{8}$ del judo y, como parte del aprendizaje, uno necesita equilibrar la "perfección de la técnica" con "la perfección de uno mismo" (0taki \& Draeger, 1983: 35-46, 58-61).

Todas las katas del Kodokan reflejan un objetivo ideal del judo -"buen uso de la mente y el cuerpo"- a través de la transmisión de conceptos como la correcta acción/reacción, sacar partido de un momento de oportunidad (debana), ruptura del equilibrio (kuzushi) y la "mente presente" (zanshin). De manera adicional, cada una de las katas del Kodokan realiza una contribución específica a la enseñanza de diferentes principios y aspectos de control inherentes al judo - "por ejemplo, control de la respiración, control de la postura y del equilibrio, control de la velocidad, control del movimiento del cuerpo (tai-sabaki), de la sincronización y, de manera primordial, control emocional-. Incluso la Koshiki-no-kata y la Itsutsu-no-kata, que parecen en primera instancia ser muy antiguas comparadas con el judo actual, tienen su valor. La comprensión que puede derivarse de su correcta práctica contribuye de modo importante al desarrollo del "sentir interior" (kimochi), del "sentido de la ruptura del equilibrio" y del "sentido de posición y del posicionamiento" (tsukuri).

En el libro Judo, que marcaba el centenario del nacimiento de Kano Jigoro, los representantes del Kodokan declararon: "Cada... kata, a la vez que muestra cierto número de movimientos clásicos, posee un profundo significado. Cuanto más se practican las katas mayor es la consideración de las mismas como bienes culturales que encarnan la esencia del Judo" (Kodokan, 1961:65).

\section{Mirando con mayor profundidad las katas del Kodokan}

A cada kata del Kodokan la recorre su propio hilo o tema subliminal. Sin embargo, cuando uno empieza a aprender una kata particular, simplemente se están aprendiendo sus movimientos mecánicos superficiales. De hecho, no es hasta mucha práctica y auto-exploración posterior que se revelan los principios y temas incluidos en la kata tal y como se aplican en la práctica del judo. Las siguientes páginas analizarán con mayor profundidad cada una de las katas del Kodokan para facilitar el desarrollo de una comprensión más detallada.

\section{Formas de práctica libre}

De forma conjunta, la Nage-no-kata y la Katame-no-kata son conocidas como Randori-no-kata (formas de práctica libre). Proveen un marco que facilita el desarrollo de las habilidades de práctica libre, y es esencial que sean practicadas

\footnotetext{
7 La práctica de cualquier kata, sin duda, contribuye de manera importante al desarrollo del refinamiento técnico, ya que para hacer kata uno debe ser capaz de "tener la sensación" (o "sentir") al compañero. Es posible no obstante ganar en práctica libre y competición sin "sentir", usando principalmente los elementos de sorpresa, velocidad y fuerza. Esos elementos son aspectos del judo efectivos y muy importantes, pero no representan el judo en su globalidad. Más aun, están abocados al fracaso debido a que uno siempre puede encontrar a un oponente que es más rápido y más fuerte.

8 Aquí la comprensión no se refiere tan sólo a la parte intelectual, sino también a la comprensión de los principios y a la comprensión corporal.
} 
con la actitud que uno tendría en práctica libre, i.e., con entusiasmo y compromiso, y desde luego sin "pretensión teatral".

Se explicará ahora cómo el aprendizaje que puede obtenerse de la Nage-nokata transciende el mero desarrollo de habilidades para las técnicas de proyección. Para este propósito se considerará el primer grupo de la kata, denominado "técnicas de mano". La secuencia de técnicas es "caída flotante", seguida de "proyección cargando en la espalda" seguida de "rueda por los hombros" (uki-otoshi $\rightarrow$ seoi-nage $\rightarrow$ kata-guruma).

Para beneficiarse de una explicación más extensa, creemos apropiado introducir ahora dos términos comunes en el judo: tori y uke. Durante la práctica controlada, tori es la persona que realiza una proyección u otra técnica. U ke es la persona que recibe la técnica.

- Caída flotante (uki-otoshi): cuando uke ataca a tori lo hace con toda su fuerza, sin tener en cuenta su situación, y es proyectado con una caída flotante. Uke no ataca de nuevo a tori de ese modo falto de preparación y aprende de cada ataque fallido. Más aún, tori se adapta a cada nuevo ataque.

- Proyección cargando en la espalda (seio-nage): a continuación, uke ataca a tori con un golpe descendente explosivo a la coronilla. Tori recoloca su cuerpo y dirige esa fuerza, alejándola de su cabeza y llevándola hacia el suelo, utilizando una proyección cargando sobre la espalda. De ese modo, tori permite a uke lanzarse por encima del cuerpo de tori, utilizando su (de uke) propia fuerza. No se realiza un bloqueo; tori simplemente gira y proyecta. El propósito de esta técnica es el demostrar un principio fundamental en judo (el de la no resistencia) con uke siendo anulado por la fuerza de su propio ataque. Tori no proyecta en el sentido convencional del término, sino que simplemente utiliza la propia acción de uke para derrotarle con "la máxima eficacia

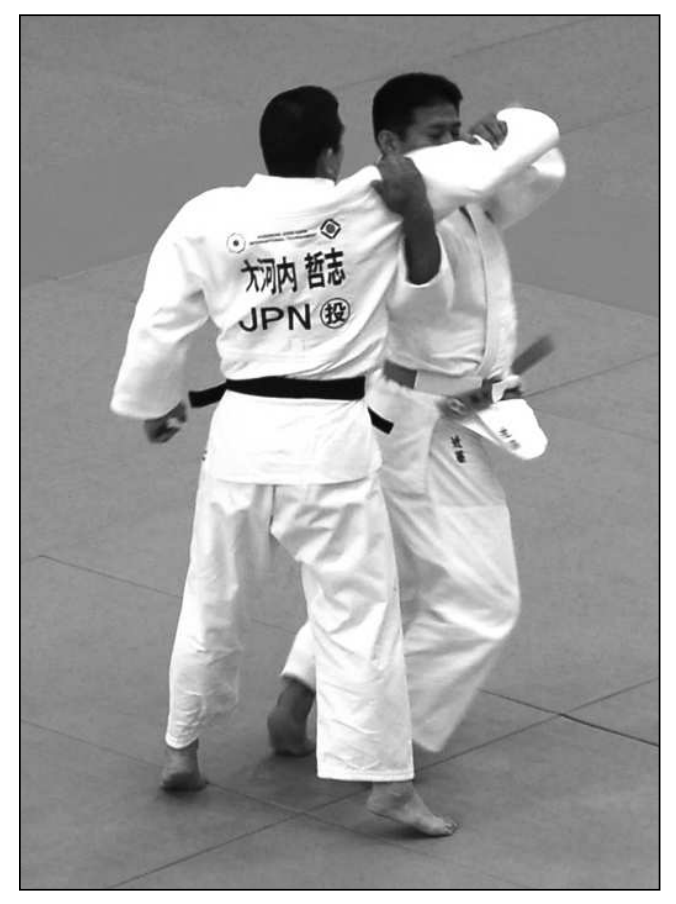
y el mínimo esfuerzo".

- Rueda por los hombros (kata-guruma): uke ataca de nuevo, pero esta vez mantiene su cuerpo rígido para evitar las respuestas de proyección que ha recibido. Una vez más, sin embargo, uke es superado por la comprensión de tori, que lo lanza con una rueda por los hombros.

Este patrón se repite a lo largo de toda la kata. En la Nage-no-kata completa, uke atacará a tori quince veces, y cada vez tori neutralizará el ataque y utilizará la fuerza de la acción del ataque de uke para dominarlo. Por tanto, a medida que tori progresa en el estudio de la Nage-no-kata, desarrolla su conocimiento de elementos biomecánicos básicos y complejos, en particular sobre cómo adaptarse para poder superar las acciones del atacante. Además, tori aprende una técnica correcta y así puede contrastarlo con aquello que aprende en la práctica libre. Finalmente, tori aprende cómo su cuerpo y mente reaccionan bajo estrés físico y psicológico, aparte de cómo adaptarse a una situación dada utilizando tanto su cuerpo como su mente de forma productiva y efectiva. 
La Katame-no-kata se refiere al aprendizaje del control, en particular a cómo tori puede utilizar su cuerpo de modo eficiente para controlar a uke en el suelo. Tori debe trabajar su versatilidad y mostrar un movimiento corporal excelente mientras lucha en el suelo, y uke debe esforzarse en escapar y tomar ventaja de cualquier debilidad en las técnicas de tori. Más aún, todas y cada una de las veces que se practica la Katame-no-kata, uke debería continuar probando a tori, buscando sus puntos débiles y atacándolos. De forma similar, tori debería encontrar nuevas maneras de neutralizar los intentos de escapar de uke. Tanto tori como uke deberían asimilar este aprendizaje en la Katame-no-kata y utilizarlo en beneficio de la práctica libre 0 de sus empeños competitivos.

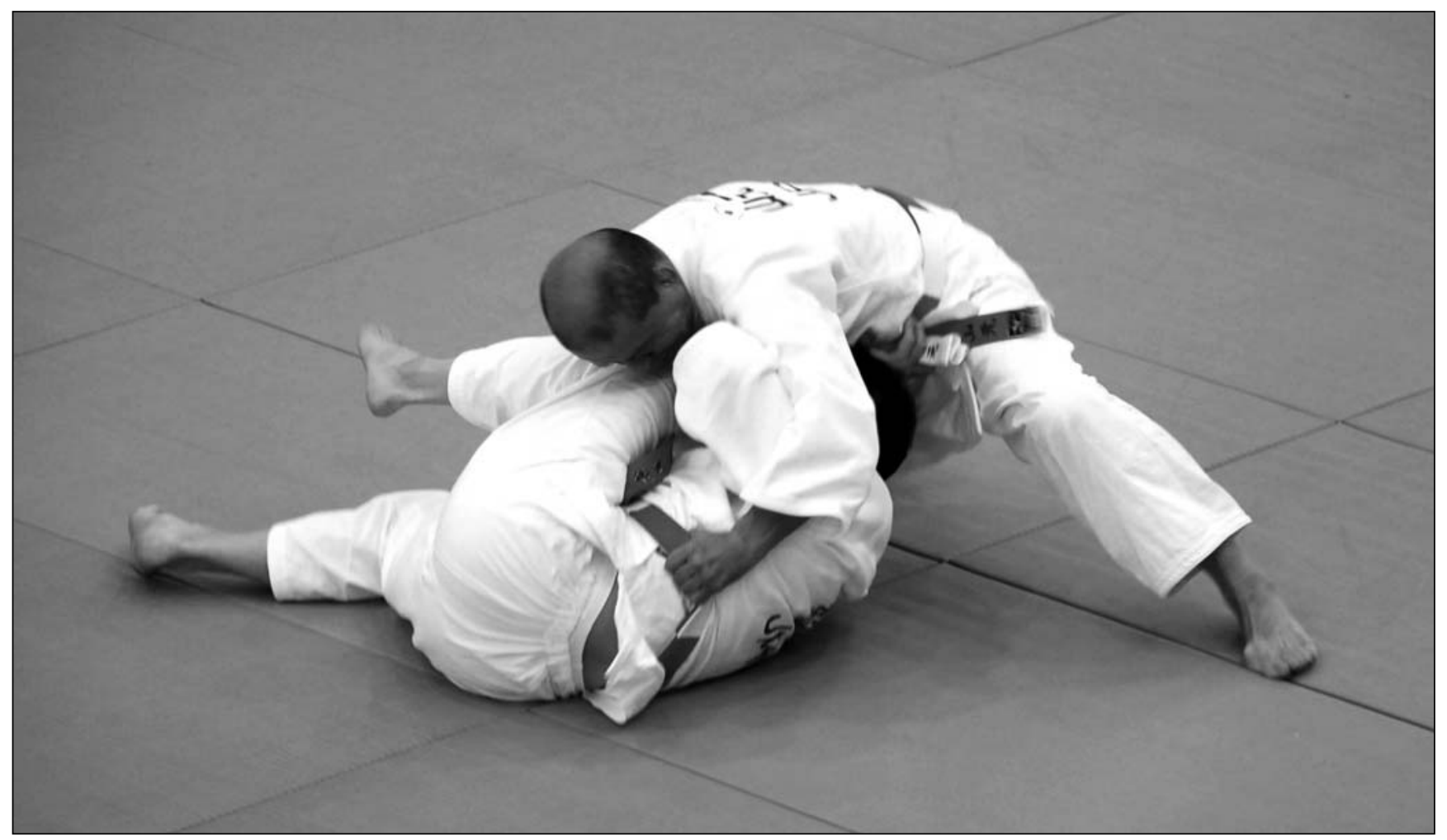

\section{Formas de defensa personal}

La Kime-no-kata, la Kodokan Goshin Jutsu y la Joshi Goshin Ho se clasifican como Shobu-no-kata (formas de defensa personal). Estas katas reflejan aquello que es crucial en una situación de lucha "real" a muerte (shinken shobu), donde el objetivo central es derrotar al adversario y sobrevivir. Enseñan movimientos corporales efectivos, velocidad, coordinación y control postural mientras se controla a la otra persona, independientemente de la técnica particular 0 de la kata estudiada.

\section{Formas de educación física}

La Ju-no-kata, la Go-no-kata y la Sei-ryoku-Zen-yo Kokumin-Taiiku se agrupan en la Rentai-no-kata (formas de educación física), donde el objetivo principal es la educación del cuerpo para que se mantenga sano. En particular, la Ju-no-kata enseña los principios de la resistencia y la no-resistencia, flexibilidad y suavidad; 
la Go-no-kata enseña el principio del uso adecuado de la fuerza controlada y desarrolla el acondicionamiento físico; y la Sei-ryoku-Zen-yo Kokumin-Taiiku favorece el desarrollo físico y la coordinación e igualmente pone los cimientos para el desarrollo de las habilidades básicas del judo.

La Ju-no-kata no enseña "técnicas de lucha" en sentido convencional o con la idea de utilizar de modo literal esas técnicas en combate. En vez de eso, la Juno-kata incluye una serie de ataques y defensas para demostrar la eficiente redirección de la fuerza y del movimiento. Además, como ni tori ni uke son proyectados 0 controlados en el suelo durante el transcurso de la kata, puede ser practicada en cualquier sitio y por cualquiera, incluso por aquellos que son ancianos o de carácter nervioso. La Ju-no-kata transmite el principio de no contrarrestar fuerza con fuerza; enseña cómo responder a la acción con una reacción que, en lugar de utilizar la fuerza (go), lo hace cediendo (ju). U ke ataca a tori y tori neutraliza el ataque, utilizando el ataque mismo para vencer. Por esta razón, aquellos que practican la Ju-no-kata solo como quince movimientos corporales están perdiendo el sentido de la kata. Aunque el elemento físico de la kata ofrece lecciones vitales sobre la acción de ceder, la Ju-no-kata es realmente un conjunto de ejercicios en los que tori controla su mente y emociones para encontrar su armonía interior y luego mezclar dicha armonía con uke. La Ju-no-kata por tanto hace honor a su nombre y es considerada por muchos como la mejor de todas las katas del Kodokan.

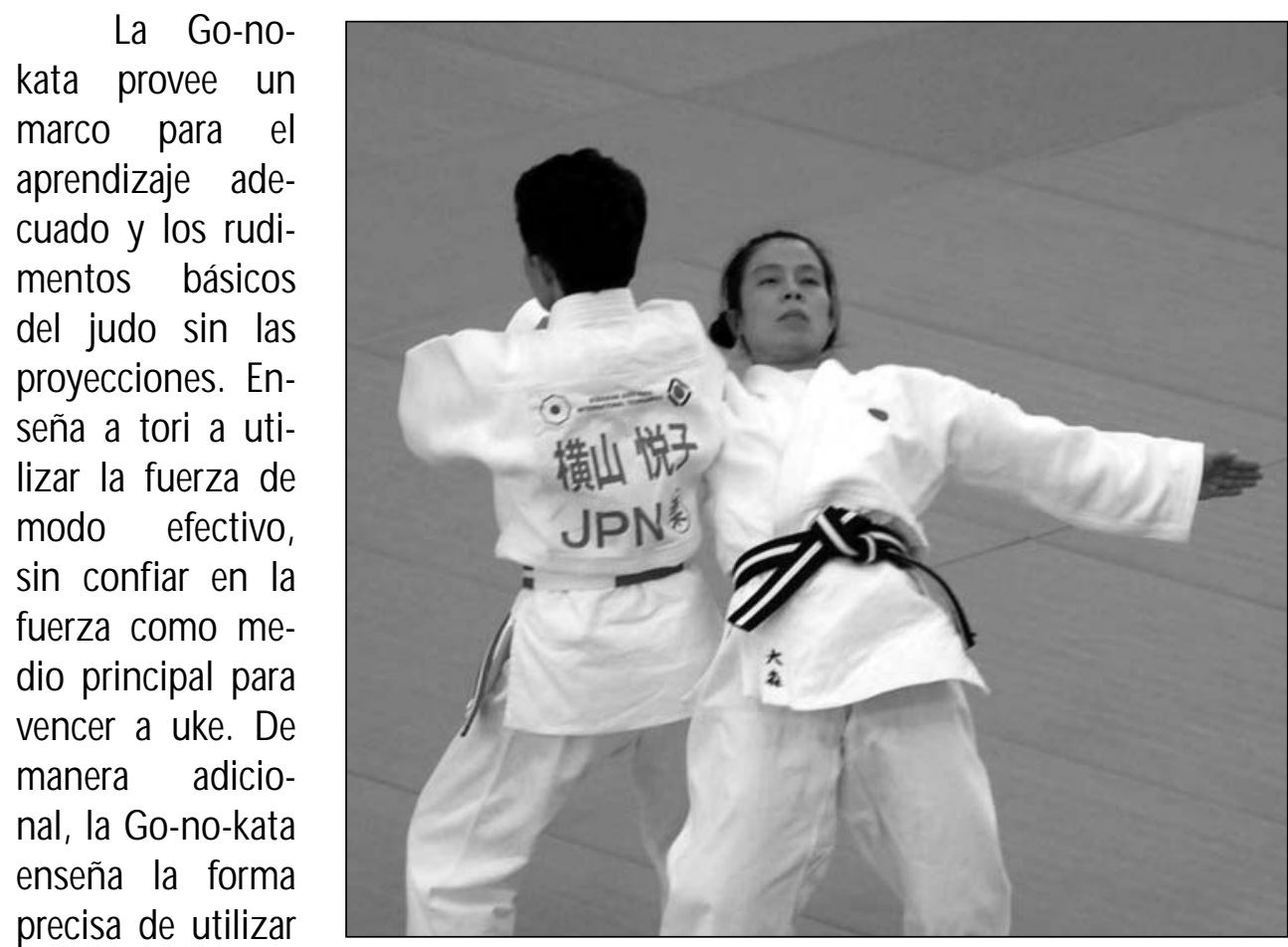

el propio cuerpo,

especialmente cómo usar tanto la fuerza focalizada como el ceder en momentos críticos durante las técnicas de judo. La Go-no-kata ayuda además al desarrollo de la fuerza física en sí y contribuye a incrementar la fuerza de voluntad y la "fuerza espiritual" en el sentido de "un espíritu saludable en un cuerpo saludable" (De Crée \& Jones, 2009). 


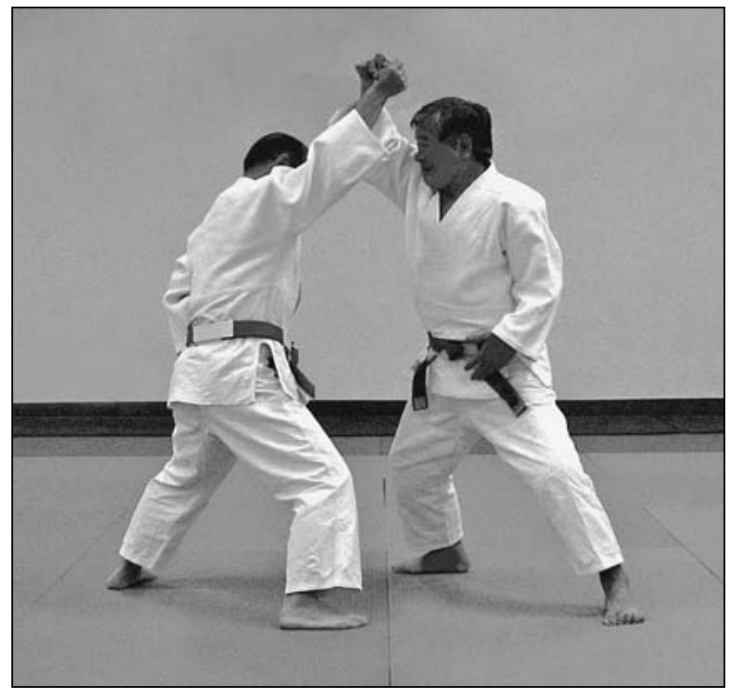

Tanto la Ju-no-kata como la Go-no-kata transmiten el sentido de ju-no-ri, i.e., el principio central del jujutsu por el cual uno evita oponerse directamente a la fuerza y potencia del contrario, sino que la utiliza en favor de la propia ventaja. Además está de acuerdo con un principio básico del judo, que puede ser expresado de distintos modos: "la suavidad vence a la dureza", "la flexibilidad vence a la rigidez", "la delicadeza controla a la fuerza" o "vencer cediendo" (Ibid).

La Sei-ryoku-Zen'yo Kokumin-Taiiku no es estrictamente una kata, sino más bien un ejercicio de educación física ${ }^{9}$ que puede practicarse de manera muy informal con un considerable grado de libertad (nótese además que la palabra "kata" ni siquiera aparece en el nombre, reflejando que es en sí de naturaleza más libre que las series denominadas ho o "método"). Se divide en dos tipos de ejercicios: práctica en solitario (tandoku renshu) y con compañero (sotai renshu).

Los ejercicios en solitario contienen golpes y patadas, así como varios ejercicios diseñados para desarrollar el tono muscular y un físico equilibrado. Normalmente, cada movimiento se repite cinco veces, izquierda y derecha, antes de continuar con el siguiente. Sin embargo, hay libertad para aumentar el número de repeticiones si se desea.

Los ejercicios con compañero se dividen en dos grupos principales: Kime Shiki (formas de decisión) y Ju Shiki (formas de suavidad). Las técnicas de Kime Shiki contienen versiones sencillas de muchas de las técnicas encontradas en la Kime-no-kata, incluyendo agarres, golpes y ataques con armas. La parte de Ju Shiki contiene dos grupos de cinco técnicas cada uno, tomados directamente de la Ju-no-kata.

\section{Formas de teoría}

La Itsutsu-no-kata y la Koshiki-no-kata se agrupan en la Ri-no-kata (formas de teoría). Ambas katas se enraízan en las escuelas tradicionales de jujutsu y transmiten principios de estas escuelas de origen. No obstante, debería tenerse en cuenta que estos principios son desconocidos para la mayoría ${ }^{10 ;}$ no obstante,

\footnotetext{
9 A pesar de los enormes esfuerzos de Kano para la promoción de la Sei-ryoku-Zen-yo KokuminTaiiku como una kata de ejercicio físico, no fue apreciada en la medida que él hubiera deseado, en parte por el momento de su muerte y en parte por la disrupción causada por la Segunda Guerra Mundial.

10 A excepción de algunos de los profesores más veteranos del Kodokan, ningún profesor japonés u occidental es capaz de explicar en su totalidad la esencia y aplicabilidad de la Itsutsu-no-kata y de la Koshiki-no-kata y cómo estas se integran en el extenso programa del judo.
} 
cabe decir que algunos conceptos, como el de "mente inamovible" (fudoshin), son algo conocidos en la actualidad.

La Itsutsu-no-kata expresa los principios de ataque y defensa en movimientos que evocan fenómenos naturales y pueden considerarse como manifestaciones físicas de la "mente filosófica" del judo. Discutiendo la Itsutsu-no-kata en la publicación Judo (1961), los representantes del Kodokan afirmaban: "Es una kata artística y llena de sentido que busca que los movimientos naturales (el movimiento del agua, el movimiento de los astros celestes, etc.) sean habilidosamente expresados con el propio cuerpo" (Kodokan, 1961:67).

De este modo, la Itsutsu-no-kata puede considerarse como la kata de los "principios de movimiento", una especie de "física del judo". Aunque la Itsutsu-no-kata se atribuye oficialmente a Kano Jigoro (Kano, 1986), investigaciones recientes de De Crée (2010) indican que no fue creada por Kano, y que existía claramente en la Tenjin Shin'yo-ryu jujutsu bajo el nombre "Enseñanzas orales en cinco pasos" (Kuden Gohon).

La Koshiki-no-kata se introdujo en el repertorio del Kodokan como recordatorio de que la herencia del judo se situaba parcialmente en el jujutsu de la Kito-ryu, en particular en el estilo Takenaka-ha (Ibid). De acuerdo con esto, la Koshikino-kata es conocida también como la Kito kata y puede entenderse como la kata de "la tradición del judo"o de la "historia del judo".
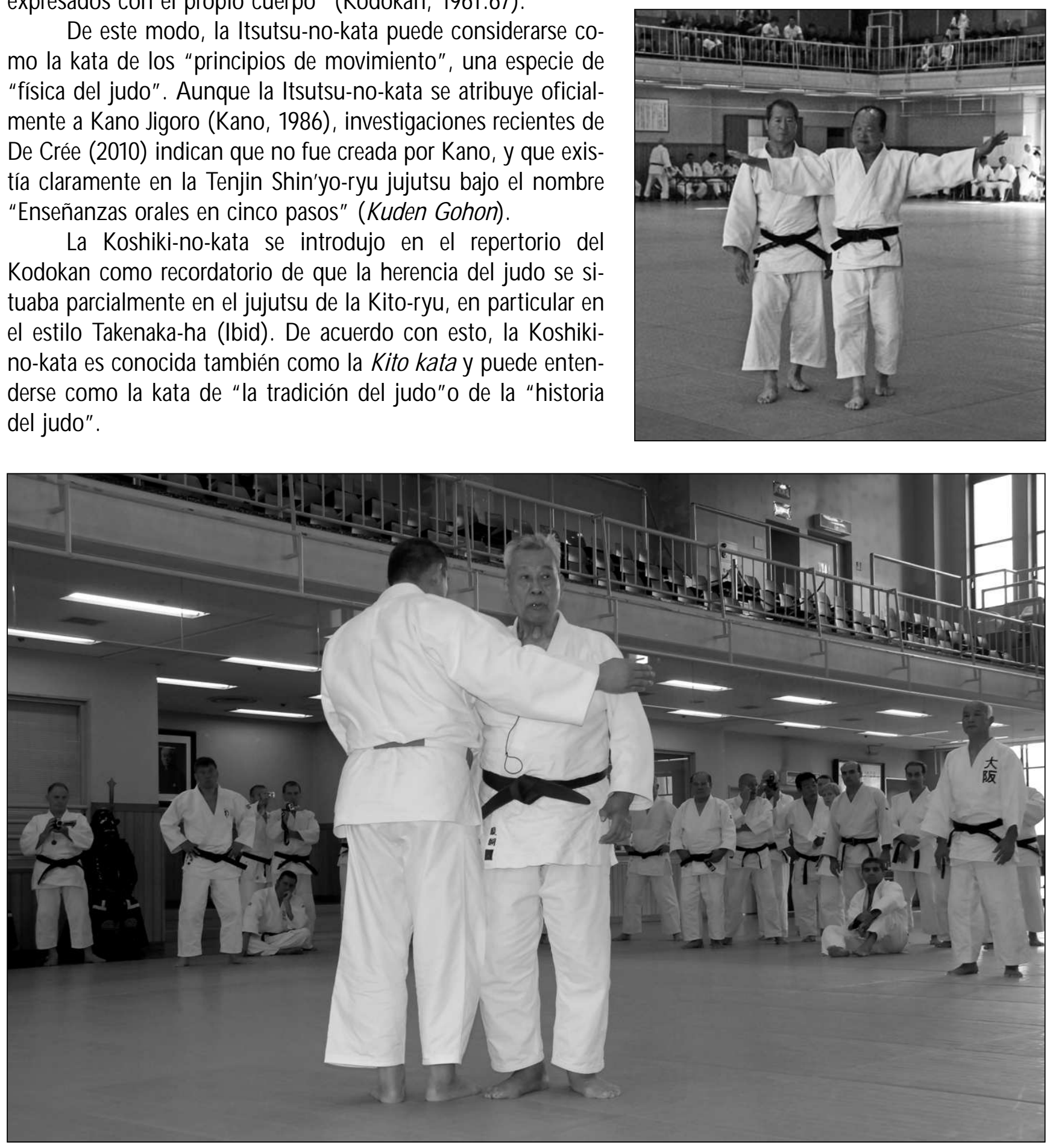
De acuerdo con el Kodokan (1961), el primer movimiento de la Koshiki-nokata, la "postura de preparados" (tai), es la base de toda la kata: "Tai es la base de la Kito kata. Enseña que es importante adoptar una postura calmada, aquietar el espíritu, despejar la mente y, de ese modo, discernir las fortalezas y debilidades del oponente. Así pues el objetivo original de la Koshiki-no-kata era enseñar no tanto técnicas como métodos de entrenar la mente" (Kodokan, 1961:69).

El propósito central de la Ri-no-kata es desarrollar un entendimiento más elevado de la "esencia" fundamental y más profunda y quizá de los principios "esotéricos" del judo (De Crée \& Jones, 2009). La Ri-no-kata introduce el estado mental del atacante y los principios de la física, la anticipación y la reacción. Requiere desarrollar una comprensión del estado mental del atacante en el momento del ataque ("¿Qué está tratando de conseguir?" y "¿Cómo se anticipa uno al ataque?") y de qué leyes de la física y principios del ceder deberían aplicarse a cada situación. Estas son ideas muy complejas y es la síntesis y la comprensión de todos esos conceptos en las katas individuales que hacen que la Itsutsu-no-kata y la Koshiki-no-kata sean tan difíciles y estén más allá de la comprensión de la mayoría de los practicantes de judo a lo largo de su trayectoria.

La Ri-no-kata es por tanto la etapa lógica final en el proceso de aprendizaje de las katas. No pueden ser estudiadas de modo apropiado hasta que se dominen las Randori-no-kata, las Shobu-no-kata y las Rentai-no-kata.

\section{Sentimiento interior}

Ya se ha explicado cómo la kata en judo implica la síntesis de un grupo de conceptos y disciplinas físicas y mentales en la realización de rutinas y ejercicios extremadamente desafiantes y cómo eso hace del estudio de las katas una educación completa en sí misma. "El concepto de kata a menudo no se entiende correctamente. Aunque se realicen varias técnicas, la kata no debe considerarse como un catálogo de respuestas diseñadas para situaciones específicas de peligro. Más bien, la kata es un método de transmisión de principios y conceptos nucleares" (Cunningham, 2008: 10).

Por tanto, las katas se refieren al sentir interior (kimochi) y son tanto para el entrenamiento de la mente como del cuerpo. Si las katas se realizan como "meros movimientos", entonces se pierden esos aspectos educativos.

\section{Kata, energía intrínseca y no-mente}

Guy Pelletier, el practicante de judo de ascendencia no japonesa que ostenta el mayor grado Kodokan ( 8 - Dan), considera la relación de la kata, la energía intrínseca (ki) y la "no-mente" (mushin), cuando escribe:

Una buena kata es más que un número de acciones físicamente correctas. Es la actitud mental de los dos compañeros realizando el kiai, 0 la concentración del espíritu. Ahí yace la esencia de la kata. El flujo de la energía mental se deja notar claramente cuando uno presencia una exhibición de calidad.

Uke, como tori, debe mostrar su "ki" o energía mental. Su actitud externa es a menudo calmada, de vigilancia tranquila y autoconfianza. Tu ki y el de tu compañero se unen y dependen del kiai, el cual induce en retorno un estado mental llamado Muga-mushin. Esto sin embargo no se 
obtiene hasta acumular mucha experiencia. Muga-mushin significa "no yo, no mente". Es un estado de indiferencia que libera la consciencia de las acciones llevadas a cabo. En este estado, el espíritu resuelve los problemas automáticamente, por así decirlo, de ahí su importancia en la defensa personal y en el judo.

Kata es el mejor camino para [que un practicante de judo] hacer judo en un estado de ignorancia respecto a las acciones que están realizando. Un [practicante de judo] es un experto cuando alcanza este estado. La kata refina el espíritu, permitiendo llevar a cabo acciones más rápidas que a través del mismo. De este modo, el arte se convierte en un "arte sin arte".

- Pelletier, s.f.

\section{Proteger, romper, separar}

Shu-ha-ri es un concepto educativo enraizado en las artes marciales y otras artes clásicas de Japón, tales como el arreglo floral, marionetas, teatro, poesía, pintura, escultura y tejido. De acuerdo con Takamura (1986), ha sido fundamental para la supervivencia de muchas de las antiguas tradiciones de conocimiento. Shu-ha-ri describe la curva de aprendizaje hacia la maestría a través de las etapas de "proteger" (imitación/absorción), "romper" (comprensión/distanciamiento/digresión) y "separar" (consolidación/trascendencia de lo físico).

Utilizando una de las notas de una lección de Ukichi, Daigo (2008) explicaba cómo uno progresa desde la forma básica hacia una comprensión profunda de la kata utilizando este concepto:

Shu Primera etapa (proteger): la etapa de shu consiste en estudiar la lección del maestro de modo correcto. Las enseñanzas de una escuela particular deben ser estudiadas fielmente, sin ningún cambio. Esta es la etapa de imitación. Brevemente, es el modo de preservar y proteger la integridad y la dignidad de una escuela. Esta es la etapa de shu.

Ha Segunda etapa (romper): después de progresar más tras la práctica repetitiva de las katas, el estudiante comienza a preguntarse y descubrir por sí mismo. Se separa de su maestro y busca más allá de la instrucción superficial de los movimientos de la kata. Después, se aprovecha la individualidad de cada uno. La amplitud del estilo se expande. El significado de la kata se investiga más profundamente. Esta es la etapa de ha.

Ri Tercera etapa (separar): más adelante, la práctica de la kata es tratada de forma libre y espontánea. Está en el ámbito de la nueva creación que hace uno mismo. Esta es la etapa de ri.

$$
\text { - Ukichi, s.f., en Daigo, } 2008
$$

Un conocimiento más profundo de la aplicación de shu-ha-ri al estudio de las katas en las artes marciales es el que ofrece Takamura (1986) y Klens-Bigman (s.f.). El material que ahí se presenta es tan relevante para el judo como para las artes específicas consideradas por dichos artículos. 


\section{Armonía, concentración, inmersión y pureza}

En una comunicación personal con los autores, el reputado practicante de judo Paul Nogaki (60 Dan) recordaba las enseñanzas recibidas de Daigo en 1980 acerca del proceso de pensamiento que uno debería esforzarse en conseguir al practicar katas. Afirmaba que "la kata en judo consiste en aprender disciplina mental y física, conectando ambas al intentar realizar rutinas y ejercicios de extrema dificultad. Incluso las palabras para describir los objetivos últimos a obtener en las katas -in'yo-wago, senshin, seiboku y genshitsu- son casi imposibles de traducir correctamente a otro lenguaje [que no sea el japonés]" (Nogaki, 2009).

Tanto N ogaki (2009) como De Crée (2009) traducen cortésmente los conceptos introducidos por Daigo para explicar el último propósito de este "nirvana de la kata".

- In'yo-wago: In'yo-wago significa armonía entre las energías yin y yang ${ }^{11}$ (y lo que significan en artes marciales) que llevan a la fuerza. Este concepto, sin duda, tiene su origen en la tradición Kito-ryu y no en el judo. El obtener este equilibrio entre yin y yang es un largo proceso y no algo que se discuta 0 ataje de forma consciente en el judo. Podría decirse que es el objetivo de la Ri-no-kata, aunque la práctica de otras katas también puede ayudar.

- Senshin: Senshin significa literalmente "comprometido", "atención no dividida" o "concentración" "componentes importantes en el desarrollo del poder espiritual-. Sin embargo, en judo, el concepto de ki no puede cultivarse en ese sentido, si bien Kano Jigoro utilizó y habló de sei-ryoku u "optimización", el cual es su equivalente (esta fue una decisión consciente, para desmitificar el ki). Para Kano, sei-ryoku existe en dos componentes: un componente físico (chikara) y un componente espiritual (sin que sea algún tipo de poder superior místico). Usando los dos en armonía (como en in-yo-wago) se obtiene como resultado la "optimización", a menudo conocida en judo como "uso eficiente (o mejor uso) de la energía".

- Genshitsu: Genshitsu significa literalmente "compromiso". Esto puede extrapolarse para significar "inmersión total".

- Seiboku: Seiboku significa "ser delicado y puro", por tanto difiere del judo de fuerza y abrupto. La pureza se refiere a la técnica apropiada y al uso de la ruptura del equilibrio, sincronización y colocación. De este modo la fuerza no es necesaria, como el judo de "chaqueta vacía" de Mifune Kyuzo"12.

Lo anterior es una contribución original clave del presente artículo, ya que los autores no conocen otra fuente donde estos conceptos se relacionen con la práctica de la kata.

\section{Los campeonatos de katas y la deportivización de las katas}

Bajo este título se presenta una evaluación crítica del impacto que la "deportivización" de las katas está teniendo sobre este elemento tan importante del

11 En la teoría china, yin y yang: el principio fundamental de dos energías polares mutuamente interdependientes y en constante interacción que sostienen a todos los organismos vivos.

12 Mifune Kyuzo (10을 Dan) era conocido por la suavidad de sus técnicas. Era capaz de derrotar a estudiantes el doble de grandes que él sin apenas esfuerzo. La gente que entrenaba con él diría más tarde, "Era como luchar contra una chaqueta vacía". 
judo. De este modo, las preguntas realizadas por Oltremati (s.f.) serán contestadas de modo indirecto: "ipuede la práctica de las katas en judo considerarse como deporte? ¿Puede una ejecución de una forma de judo compararse a un ejercicio de gimnasia?" (Oltremari, s.f.).

\section{Historia}

Los campeonatos de katas no son algo nuevo. La British Judo Association (BJA) realizó el primer Campeonato Nacional de Katas (junto con el primer Campeonato Británico de Veteranos) en el Woolwich College de Londres en 1981. Recientemente, sin embargo, se ha producido un incremento considerable en el número y nivel de los campeonatos de katas y eventos que se desarrollan de forma regular a nivel regional, nacional, continental y mundial.

A nivel continental, por ejemplo, el primer Campeonato Europeo de Katas fue auspiciado por la BJA y se realizó en Burgess Hill, Sussex, en 2005 (al mismo tiempo se celebró un Campeonato de Veteranos). El evento, al que asistió Daigo en representación del Kodokan, fue dominado por las parejas de kata italianas. Los subsiguientes Campeonatos Europeos de Katas se auspiciaron por la Unión Europea de Judo (EJU) en Italia (2006), Alemania (2007), Malta (2008) y Rumanía (2009). Otras asociaciones continentales también han organizado sus propios campeonatos de katas.

A nivel mundial, los campeonatos han sido organizados desde 1999 por la World Masters Judo Association (W MJA). La W MJA es una entidad fundada en Ottawa, Canadá, en 1998 con el propósito de motivar la participación en el judo competitivo y en la práctica de katas para aquellos practicantes de judo con treinta 0 más años, en un ambiente de diversión, amistad y familiaridad. El primero de estos eventos fue realizado en W elland, Canadá. Subsiguientes campeonatos de katas (y de judo competición) de la W MJA se han celebrado en Canadá (2000), EEUU (2001), Reino Unido (2002), Japón (2004), Canadá (2005), Francia (2006), Brasil (2007), Bélgica (2008) y EEUU (2009).

La propia IJF fue algo lenta en la organización de campeonatos de katas, teniendo lugar el primer evento de la IJF, conocido como Copa Mundial de Katas, en París, Francia, en noviembre de 2008, siendo esencialmente un ensayo para el primer Campeonato Mundial de Katas oficial de la IJF, que tuvo lugar en la Valeta, Malta en 2009. El segundo evento de la IJF tuvo lugar en Budapest, Hungría, en mayo de 2010.

Paralelamente a la organización de su propio evento de veteranos, la IJF realizó diversas actuaciones que podrían interpretarse como esfuerzos conscientes para marginalizar y asfixiar los esfuerzos de la W MJA ${ }^{13}$. En 2009, la W MJA finalmente cedió a lo inevitable y aceptó disolverse en 2010. El último evento de la W MJA fue en Montreal, Canadá, en agosto de 2010, tras lo cual el control del judo de veteranos a nivel mundial pasó a manos de la IJF. Sólo el paso del tiempo

13 En el 2009, la IJF y la EJF retiraron el permiso a sus árbitros y jueces de katas para poder oficiar eventos de la W MJA. Además, Budapest, el lugar originariamente diseñado para la celebración del evento de la W MJA en 2009, retiró su candidatura casi al final, alegando recibir presiones de la IJF. 
revelará si la IJF será capaz de preservar el espíritu de judo de veteranos tal y como originalmente lo concibió y estableció la W MJA.

El primer Campeonato Internacional de Katas de Judo Kodokan, organizado bajo los auspicios conjuntos del Instituto de Judo Kodokan y la Federación Japonesa de Judo, tuvo lugar en octubre de 2007, en el Instituto de Judo Kodokan de Tokio. Este significativo evento ha sido descrito por Gatling (2008).

\section{Campeonatos de katas: lo positivo}

Está claro que la introducción del elemento competitivo ha otorgado al competidor de judo una fuente de motivación para estudiar las katas, i.e., el objetivo tangible de una medalla. Debe también afirmarse que algunas de las ejecuciones logradas en las competiciones de katas son sobresalientes en todos los aspectos, particularmente las realizadas por los participantes de Italia, Japón y España. Tales ejecuciones llenas de energía son sin embargo la excepción a la regla.

Está claro también que una consecuencia directa de los campeonatos de katas es que en la actualidad hay más practicantes de judo que son conscientes de ellas y que las practican más que en cualquier momento del pasado reciente. Sin esta recuperación ligada a la competición, hay una gran probabilidad de que la práctica de las katas se hubiera desvanecido de la práctica del judo lenta y progresivamente, dejando tras de sí únicamente el poco digno y cada vez más comercializado "deporte de lucha con chaqueta" en que la IJF ha convertido al judo.

Teniendo en cuenta lo dicho anteriormente, a menudo se afirma que "Sin los campeonatos de katas, las katas estarían muertas". Este artículo ahora sostendrá que debido a los valores y constricciones impuestas por los campeonatos de katas, la kata como ayuda en el aprendizaje tal y como la concibió Kano Jigoro está ya "casi muerta". Oltremari (s.f.) escribe: "El mismo barbarismo que se ha introducido en el shiai [competición] y que lo ha convertido en un infierno realizado en una cancha está a punto de llegar a las katas. Un nuevo gimnasio para el triunfo del ego está a punto de surgir, mientras que el esquema [kata] creado para propósitos completamente distintos desaparece lentamente La introducción de los principios del deporte moderno en esta disciplina [kata] distorsionará su esencia y significado, primero condenándola a su contextualización cultural y después dirigiéndola hacia su absoluto final" (OItremari, s.f).

\section{Campeonatos de katas: lo negativo}

Se explicará ahora cómo los campeonatos de katas están erosionando los beneficios subyacentes y principios fundamentales del entrenamiento de las katas y por qué el concepto mismo (i.e., la deportivización de una herramienta educativa) debería ser considerado como algo fallido de manera fundamental.

Los campeonatos de katas no se acomodan a la individualidad, no dan cabida a la interpretación y limitan de modo rígido cualquier esfuerzo creativo en la ejecución de cualquier kata en particular. Consideran tan solo cuánto de bien se adecúa la "actuación de la kata" ${ }^{14}$ a un esquema de puntuación dado, sin tener en

14 Con respecto a las katas, el término a menudo utilizado de "actuación" (performance) es algo problemático para los autores. Actuación implica una actividad hecha para entretener 0 agradar a los demás. Como ya se ha explicado, la kata se hace para el beneficio y la educación de los dos 
cuenta el impacto que esto tiene en el desarrollo de los ejecutantes como practicantes de judo. El impacto de esto es que para tener éxito en las competiciones de kata, los practicantes de katas están obligados a entrenar, no para aumentar su comprensión o por su crecimiento personal o para mejorar su práctica de la kata, sino más bien para presentar "calcos" de los movimientos físicos de la kata tal como se muestran en las filmaciones didácticas relevantes. El único propósito de esta farsa es el permitir a los jueces llevar a cabo evaluaciones "comparando punto por punto", siendo los vencedores aquellos ejecutantes que pueden mostrar la "copia de la kata" o "clon de la kata" más atractiva a la vista, sin tener en cuenta el espíritu y realismo de la misma. A fuerza de utilizar este enfoque, los campeonatos de katas se centran sobre todo en el aspecto shu ("proteger") del estudio de la kata, y no prestan atención a los elementos ha ("separar") o ri ("abandonar").

Si el foco de la práctica de las katas se dirige hacia el éxito en los campeonatos en vez de hacia la mejora personal, entonces todo el entrenamiento se convertirá en algo desequilibrado debido al excesivo énfasis en aspectos técnicos menores y convenciones ${ }^{15}$ que no deberían ser fuente de preocupación en el entrenamiento regular. M ediante la copia servil de movimientos ritualizados capturados en una filmación didáctica, tanto la intención educativa de la práctica de las katas como el aprendizaje holístico asociado que resulta de su adecuada práctica se pierden. Las interpretaciones de las katas a menudo acaban en algo vacío y falso, ya que están faltas de cualquier componente de acción/reacción significativo. En realidad, las katas nunca pueden ser copiadas y todas las ejecuciones de las katas son, por supuesto, irrepetibles. Esto se debe a que no hay dos parejas ejecutantes iguales (en términos de físico y psicología) y no hay dos circunstancias que se repitan del mismo modo, incluso entre la misma pareja.

Incluso aunque uno esté dispuesto a aceptar el principio de que las katas pueden ser evaluadas, el concepto de sumar los resultados de técnicas individuales en una kata completa para ver cómo se comparan unos ejecutantes con los demás para así ganar medallas (como en patinaje sobre hielo o gimnasia) basado en este ranking, es absurdo. Cada kata es una representación completa desde el principio hasta el final, y cualquier evaluación de las katas debe ser por tanto en su conjunto y no como suma de sus partes individuales.

La absurdez del enfoque de la "suma de las partes individuales" para evaluar la ejecución de las katas puede ilustrarse en el ejemplo de Daigo, que estaba demostrando la Nage-no-kata en la "Ceremonia del corte del pastel de arroz de Año Nuevo" en el Kodokan (Otaki \& Draeger, 1983:432). Daigo fue víctima de un

practicantes de judo que hacen la kata. Cualquier juez que entienda verdaderamente las katas debería fácilmente ser capaz de diferenciar entre una pareja de practicantes de judo "haciendo" genuinamente la kata de la pareja que tan solo está "actuando".

N.d.T: el término inglés performance puede traducirse al castellano como "actuación" o como "ejecución". Considero que en este caso, si bien el término más común en nuestro idioma sería "ejecución", la traducción con el término "actuación" transmite de modo más adecuado aquello que los autores quieren expresar aquí.

15 La IJF ha producido una "fórmula competitiva" (IJF, 2010; 2010a) que detalla cuántos puntos han de reducirse por categorías de "errores técnicos" en la ejecución de los campeonatos de katas. Consecuentemente, cuando practican, los competidores de judo enfatizan evitar tales errores en detrimento del espíritu mostrado o el realismo de la kata. 
error de su uke, que le atacó con una técnica fuera de la secuencia, empezando el cuarto grupo ("técnicas de sacrifico de frente", ma sutemi waza) con un golpe en vez de acercarse y realizar un agarre como prescribe la proyección correcta en la secuencia, la "proyección en círculo" (tomoe-nage). Daigo reaccionó sin fisuras y con efectividad: no pudiendo realizar el agarre prescrito, simplemente hizo la entrada y ejecutó la respuesta lógica al ataque, la "proyección hacia atrás" (ura-nage). Tras esto, realizó el lanzamiento a la izquierda. Seguidamente realizó tomoe-nage (como segunda técnica) y posteriormente continuó realizando la tercera correctamente. Tal era la intensidad de las acciones de Daigo, y estaban tan bien coordinados sus reflejos y reacciones, que muchos espectadores no se dieron cuenta de lo que había ocurrido.

La respuesta de Daigo fue la mejor posible en esas circunstancias y fue mucho mejor que reaccionar con dudas, pararse por completo 0 empezar de nuevo. Sin embargo, en el contexto de la Nage-no-kata, la ejecución global contiene un "error". La pregunta entonces se centra sobre las consecuencias de un "error" en la kata. En una competición de katas, un error como el realizado por el uke de Daigo se habría visto como algo "muy pobre" y habría resultado (como mínimo) en una puntuación de cero (IJF, 2010; 2010a) para esa técnica en particular. En una competición de katas eso habría significado que no habrían tenido ninguna posibilidad de medalla.

Sin embargo, cuando lo ocurrido se evalúa en el contexto más amplio del judo, el error puede considerarse como algo trivial, solo siendo un error en la progresión lógica de la Nage-no-kata. Daigo debería ser felicitado por evidenciar una mente flexible y por ejecutar una respuesta adecuada contra ese ataque particular. El gran judo no se refiere a cómo uno ejecuta bajo circunstancias favorables, sino a cómo uno ejecuta, actúa y responde bajo condiciones de riesgo o no anticipadas.

Resulta particularmente descorazonador para los que quieren promover un estudio serio del judo y sus katas el hecho de que, como consecuencia directa del énfasis que se pone en la actualidad en la competición, el verdadero propósito y lugar de la práctica de las katas de judo como herramientas de aprendizaje se esté perdiendo. Además, se está promulgando información necia o incorrecta sobre las katas, siendo un ejemplo especialmente atroz la descripción sin valía alguna que la directora de comunicación y relación con los medios del Judo de EEUU (el Órgano Nacional de Gobierno en el judo de EEUU), N icole Jomantas realiza: "Kata es una disciplina del judo basada en la forma en la cual dos atletas realizan una rutina de técnicas de judo que son puntuadas por unos jueces" (Jomantas, 2009).

La definición de Jomantas refleja la desafortunada actitud hacia las katas que impregna el judo contemporáneo, i.e., la obsesión de los Ó rganos $N$ acionales de Gobierno con la competición, opuesta a los aspectos educativos del judo. Es además patente que Jomantas no ha pensado en la lógica de su propia definición, ya que una consideración rápida de la cuestión "Si una kata se realiza sin jueces, ¿no es aun una kata?" hubiera puesto de manifiesto lo errónea de aquélla. 


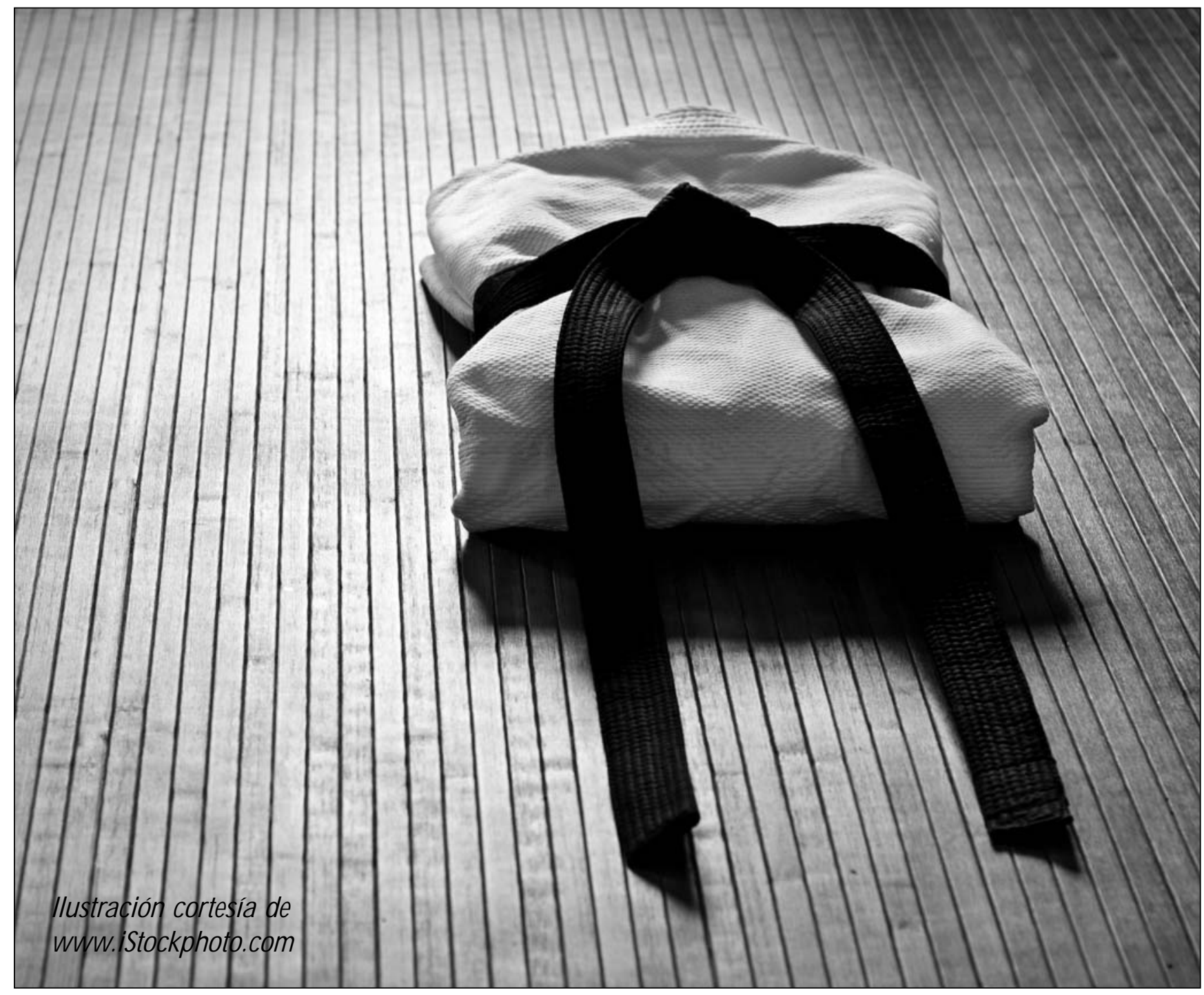

\section{judo}

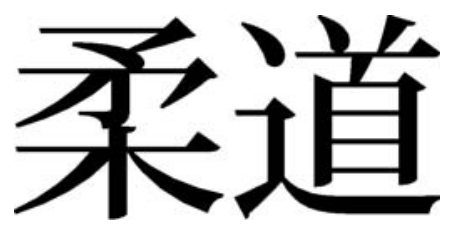

\section{"el camino flexible"}




\section{Poniendo en cuestión el propósito de la práctica de las katas}

Las katas no fueron creadas para ser "juzgadas" o para ser estudiadas como un ejercicio de "cómo realizar una demostración ceremonial de katas"; más bien, fueron desarrolladas como herramientas vivientes y llenas de energía para apoyar el correcto aprendizaje y práctica de los principios del judo.

Tal y como las concibió Kano, las katas tenían una intención principal como tipo de práctica. No obstante, cuando alguien ve katas hoy en día, siempre es como parte de una exhibición y casi nunca como una práctica real o como técnica de defensa personal, o simplemente como ejercicios previos o posteriores al entrenamiento. En los tiempos de Kano, la práctica de las katas no necesitaba ser una ejecución ceremonial completa, sino simplemente una actividad para mejorar la comprensión que uno tenía del judo, o para calentar, o para fortalecer los músculos, o para mejorar los reflejos, la flexibilidad, la coordinación, la concentración o para aprender realmente cómo defenderse ante un cierto ataque.

Cuando se estudian las katas inicialmente, es fácil implicarse demasiado en su ceremonia, ritual y mecánica. A pesar de la genuina importancia de estos aspectos, no se les debe permitir que desmerezcan sus mayores lecciones. El comprender esas lecciones es necesario para que los practicantes se cuestionen y pregunten "¿Qué es una kata?" con mayor profundidad y con una mayor comprensión y apreciación. Mientras que el entrenamiento para una competición de katas ayudará al aprendizaje de movimientos técnicos específicos de la kata, no conducirá sin embargo a una comprensión de por qué uno estudia katas, de sus beneficios, de las intenciones de los creadores de las katas 0 de la tradición y psicología de la práctica de las katas.

Las katas deberían ser estudiadas siempre por su contenido educativo y nunca solo para que "parezcan bien". Las katas pertenecen a cada practicante de judo como individuo y no pueden ser el clon de ninguna otra persona. De este modo, todas las interpretaciones de la kata deberían ser diferentes, y a un nivel elemental, para que la interpretación de la kata sea "correcta", tan sólo el orden de las técnicas, el protocolo de la kata en cuestión y un espíritu apropiado son las cosas esenciales a preservar.

Contra el propósito y meta original de las katas, está claro que la única justificación de los campeonatos de katas es la posibilidad de poder recibir críticas por parte de los practicantes de igual grado y veteranos. Sin embargo, la retroalimentación de los iguales y de los de mayor grado debería poder obtenerse en cualquier tipo de competición en la que participemos con otros. De hecho, el concepto de competir contra otros en la realización de katas debería ser un anatema para cualquiera incluso sólo con los conocimientos más rudimentarios del judo Kodokan. "¿Cómo puede uno competir en un ejercicio que trata sobre el desarrollo personal?" "¿Cómo puede uno juzgar a un ser humano frente a otro?" $Y$ ya que el judo es la educación del ser humano físico y emocional, "¿Cómo se puede llegar a emitir tales juicios (ya que la práctica de las katas de cada uno es una manifestación personal del judo y por tanto de uno mismo)?".

Las katas que se realizan bien y con comprensión pueden ser un espectáculo emocionante e interesante para un observador, así como entretenido y gratificante para los participantes. Las exhibiciones mecánicas de katas clonadas 
no tienen ninguna valía ni para los participantes ni para los observadores. Como se ha explicado anteriormente, el espíritu y la actitud mental de una persona que realiza la kata dicta su sentir y su cadencia, y esto puede ser tan personal como cualquier otra cosa que se realice en judo. Como afirma Damblat: "Aprender una kata es relativamente fácil, pero conocer una kata es tan sólo el primer paso. El objetivo último es descubrir, comprender o captar su raison d'etre y el mensaje que transmite. Uno primero debe asimilar, después sentir y eso [la kata] debe ser algo vivo. La ejecución de la kata no debe nunca realizarse de forma mecánica y sin alma. No tiene por qué ser demostrativa, sino que simplemente debe estar viva y ser lógica mientras se muestran los principios rectores del judo para que la práctica sea beneficiosa y contribuya a algún tipo de evolución y comprensión del judo La estética de la kata debe reflejar la sinceridad del control, técnicas específicas y una comunión perfecta entre la mente de ambos compañeros" (Damblat, s.f.: 3).

En este punto, los autores darán cuenta de su desilusión con el hecho de que el Kodokan haya condonado y abrazado el concepto de los campeonatos de katas. Aunque Kano Jigoro reconocía el valor de las competiciones, las katas utilizadas como competiciones sustitutas nunca formaron parte de su pensamiento. Los autores reconocen, sin embargo, que los motivos del Kodokan para actuar así eran sinceros y bien intencionados (Kano, 2007) ${ }^{16}$.

Podemos ver hoy en día un creciente interés por aprender katas, no sólo en Japón sino también en todos los países del mundo. Como resultado, el Primer Torneo Internacional de Katas del Judo Kodokan tendrá lugar en 2007... Sinceramente espero que los judokas contribuyan al desarrollo del judo a través del aprendizaje de las katas.

La mayoría de los competidores y jueces que participan en el evento están completamente implicados en el judo como instructores. Tengo grandes expectativas de que todos los participantes tomen esta oportunidad para reconocer la esencia del judo Kodokan de una forma renovada a través de las katas y así promover el auténtico judo.

A pesar de su genuina motivación para apoyar los torneos de katas, es el Kodokan el guardián del legado de Kano Jigoro, y debería ejercer esa función en parte a través del establecimiento y mantenimiento de estándares apropiados. La promoción de una kata que es falsa, vacía y sin acción/reacción no es judo, y el concepto de competir contra otros en la ejecución de katas es totalmente contrario a la filosofía e intención del fundador.

Para cerrar esta sección, merece la pena resaltar que quizás "algunas luces existen al final del túnel", debido a que algunos estudios serios se están llevando a cabo en el Kodokan, que podrían interpretarse como parte de un esfuerzo que está en marcha para atajar la cuestión de la evaluación de las katas y quizás sus recientes deterioros (Murata $\&$ Todo, 2007; 2009).

Los objetivos principales del trabajo de Murata y Todo consisten en investigar el desarrollo de un método sencillo y claro para evaluar las ejecuciones de las katas y hacer recomendaciones sobre cómo modificar los actuales sistemas.

16 Kano Yukimitsu (nacido en 1932) es el nieto de Kano Jigoro y fue el cuarto presidente del Kodokan. Dejó su cargo en 2009. 
Para ello, examinaron los métodos de evaluación europeos y japoneses con los siguientes resultados (Murata \& Todo, 2007):

1. El sistema europeo de "únicamente penalización" no es acertado ya que no reconoce los aspectos positivos, sólo los negativos;

2. El sistema japonés no permite la diferenciación de las ejecuciones de las katas entre buena y mejor, o mala y peor;

3. N ingún sistema clarifica los principios a juzgar;

4. La ausencia de un sistema de arbitraje oficial para los campeonatos de katas.

Las conclusiones formales de Murata y Todo eran las siguientes: "La existencia de las técnicas de kata se basa en los principios racionales de Tsukuri y Kake, y esos mecanismos deberían llamarse Waza no Ri (principio de las técnicas). $\mathrm{Ni}$ los estudiantes ni los profesores podrían aprender y enseñar ninguna kata sin saber W aza no Ri. Ningún juez podría evaluar la ejecución de katas sin conocer Waza no Ri" (Ibid). Tsukuri se refiere a la posición del cuerpo en la preparación para ejecutar una proyección. Kake es la finalización o ejecución de la proyección.

Murata y Todo (2009) recientemente extendieron su trabajo con una atención especial sobre la Koshiki-no-kata; este estudio aún no ha sido evaluado por los autores del presente trabajo.

\section{Conclusiones}

Vivimos en tiempos muy diferentes a los de Kano Jigoro, y a medida que hemos ido distanciándonos de ellos nuestra comprensión de muchos de los aspectos esenciales del judo se hace cada vez menor. Las katas son herramientas fundamentales de aprendizaje y para completar el judo propio, uno debe aprenderlas, practicarlas y comprenderlas. Más aun, uno debería aprovechar las lecciones que las katas enseñan tanto para la práctica libre como para la competición.

Un argumento que podría avanzarse se refiere al hecho de que una enseñanza y evaluación de las katas requiere un nivel intelectual considerable. En el judo actual (donde los cinturones son otorgados en gran medida a partir de los éxitos competitivos presentes o pasados, en vez de a partir de un conocimiento y habilidad profunda en judo), el ostentador de un alto grado pero no intelectual está por encima en la jerarquía respecto a alguien muy intelectual pero de grado menor. Esto crea problemas cuando hablamos de la enseñanza precisa de las katas.

En general, el panorama de las katas en judo es como una naturaleza salvaje con profesores llenos de buenas intenciones pero desinformados. De este modo, la mayoría de la instrucción de katas se centra en los aspectos mecánicos (movimientos, sincronización y distancia) y no dice nada sobre el sentimiento 0 las técnicas para comprender y mejorar el espíritu. Frecuentemente esto no es culpa de los profesores, ya que a menudo desconocen los propósitos subyacentes tras aquello que enseñan y por tanto no tienen conocimiento que transmitir a sus estudiantes, más allá de los movimientos físicos superficiales. 
Lo que Fraguas escribía sobre el karate-do es también cierto para el judo: "Desafortunadamente, el 95 por ciento de las personas no entienden las katas, tan sólo los movimientos externos que son irrelevantes sin su comprensión" (Fraguas, 2001:283).

Más aun, y tal y como se ha discutido en este artículo, la introducción de las competiciones de katas tan sólo ha reforzado el énfasis en la mecánica de las katas, excluyendo virtualmente todos los demás aspectos. De este modo, existe ahora una situación paradójica en la cual la práctica de las katas se está haciendo más popular entre los practicantes de base de judo, pero el verdadero propósito y espíritu de las katas está perdiéndose cada vez más.

Si uno echa la vista atrás, hacia las formas premodernas (i.e., previo a las competiciones de katas) de hacer katas, no encuentra la forma de movimiento que la mayoría de los practicantes de judo parecen creer que implican. Por el contrario, uno adquiere un ejercicio que contiene los principios del judo, los cuales, una vez entendidos y sentidos, pueden ser experimentados con el grado de "viveza" que uno quiera dentro de los confines del propio entrenamiento -por ejemplo, trabajando con hojas afiladas en la Kime-no-kata y en la Kodokan Go-shin Jutsu, o llevando una armadura tradicional (yoroi) para la Koshiki-no-kata-. Este tipo de práctica y experimentación produciría katas muy diferentes a las robóticas formas secuenciadas que se ven en la mayoría de las competiciones de katas contemporáneas.

El estudio de las katas del modo en que fueron originalmente diseñadas no se orienta a comprender la historia del judo; más bien y en primer lugar, es para hacer judo correctamente. Mientras que los buenos profesores producen buenos practicantes de judo, los grandes profesores pueden ayudar a sus alumnos a alcanzar la grandeza en la vida. La kata "real" es una de las herramientas que los "profesores reales" utilizan.

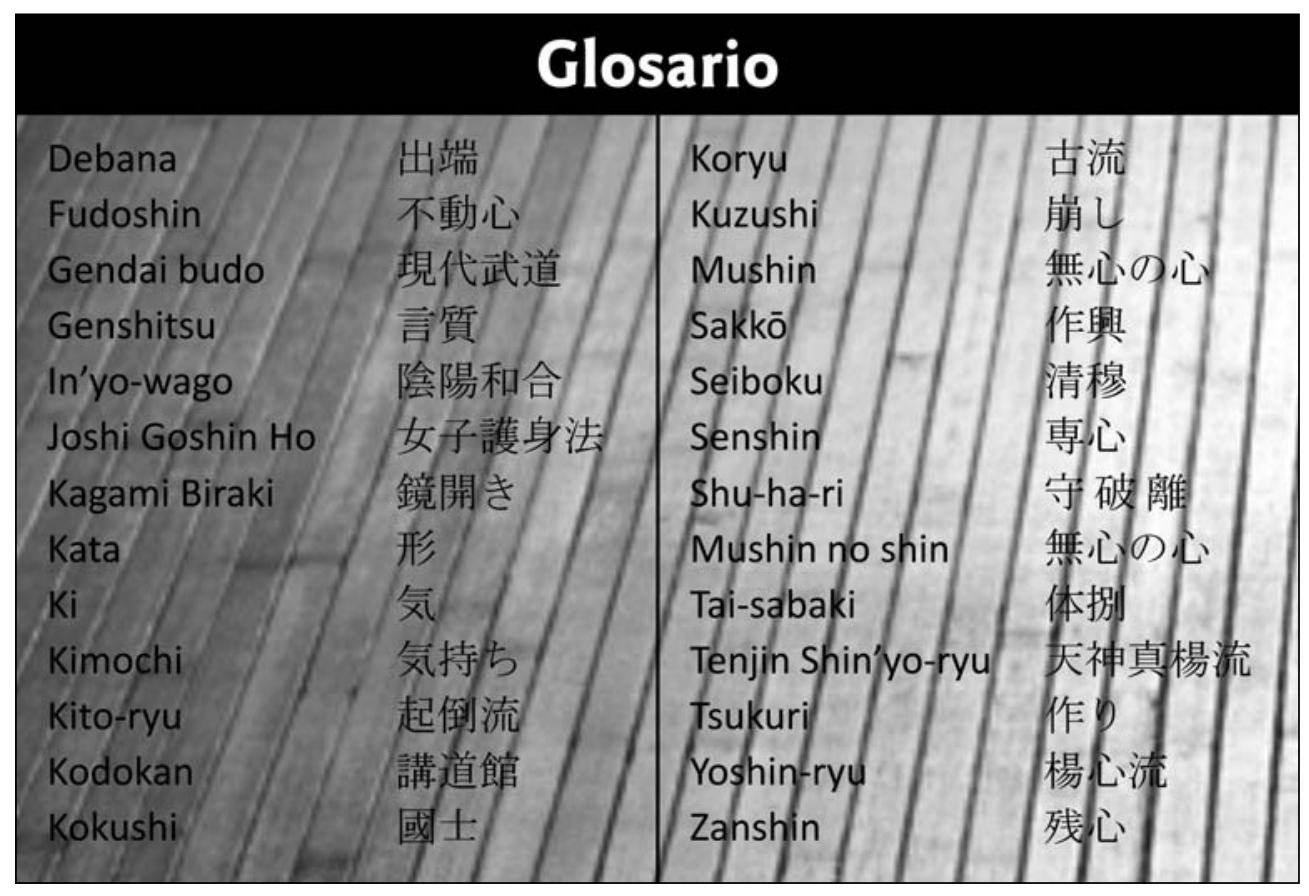




\section{AGRADECIMIENTOS}

Los autores quieren agradecer al profesor universitario Carl De Crée y a Paul N ogaki por compartir su sabiduría y conocimientos. La calidad de este artículo se ha visto enriquecida significativamente por su gran perspicacia y grandes contribuciones.

\section{NOTA}

El uso de "katas" en el artículo para denotar el plural de "kata" se ha realizado con el objetivo de estar en conformidad con el estilo editorial utilizado en la Revista de Artes Marciales Asiáticas.

\section{REFERENCIAS}

Cunningham, D. (2008). Samurai weapons: Tools of the warrior. Tokyo: Tuttle Publishing.

Cornish, J. (Primavera 2004). W hat is kata? The Bulletin, 10: 1-2. Disponible en la red y en The Kano Society. URL: http://www.kanosociety.org/ bulletins/ bulletinx10.htm (Consulta julio 2010).

Daigo, T. (2008). Jigoro Kano, Kodokan Judo-no-kata 1964. N otas de las lecciones de los Cursos de Verano sobre Katas del Kodokan. 29 Julio 2008. Tokyo: Kodokan Judo Institute.

Daigo, T. (2008-2009). Kodokan Judo Kata ni Tsuite (1-7) (Sobre las Katas del judo Kodokan, Partes 1-7). Judo, 79(10): 52-57; 79(11): 7-12; 79(12): 18-23; 80(1): 16-22; 80(2): 43-50; 80(3): $12-16$ and 80(4): 3-9. En japonés.

Damblant, R. (s.f.). Judo-Les katas ou formes: Leur histoire, leure raison d'Être et leurs valeurs. Artículo en línea disponible en Judo Quebec's W ebsite, URL: www.judo-quebec.qc.ca/img/pdf/les_kata_ou_formes.pdf. En francés. (Consulta julio 2010).

De Crée, C. (2009). Correspondencia personal con el Profesor Carl De Crée sobre cuestiones de traducción japonés-inglés en lo referente a los ideales de las katas.

De Crée, C. (2010). Ryu-setsu. Judo no musha shugyo - As snow on a willow. A pilgrimage in judo. Cambridge: Cambridge University Press (En preparación).

De Crée, C. \& Jones, L. (2009). Kodokan judo's elusive tenth kata: The Go-nokata - "Forms of proper use of force," Partes 1-3, Archives of Budo. 5, 55-73; 75-82 y 83-95. Artículos en línea disponibles en:

http://www.archbudo.com/fulltxt.php?ICID=878157 (Part 1)

http://www. archbudo.com/fulltxt.php?ICID=878158 (Part 2)

http://www. archbudo.com/fulltxt.php?ICID=878159 (Part 3)

Finn, M. (1991). Martial arts: A complete illustrated history. Leicester: Blitz Editions.

Fraguas, J. (2001). Karate masters. Burbank, CA: Unique Publications. 
Gatling, L. (2008). The First Kodokan Judo Kata International Competition and its katas. Journal of Asian Martial Arts, 17(1), 68-77.

Gutiérrez Garcia, C., Pérez Gutiérrez, M. \& Svinth, J. (2010). Judo. In Green, T. \& Svinth, J. (eds). (2010). Martial arts of the world: An encyclopedia of history and innovation, 1. Santa Barbra: ABC-CLIO.

Hicks, J. (1996). TW OJ Interviewed Trevor Leggett. Artículo online y disponible en The W orld of Judo magazine, URL: http:// www.twoj.org/archives/2000/ autumn/aut00 news5.html (Consulta julio 2010). (Originalmente en: The World of Judo (1996). Edición no 6).

Hoare, S. (2000). Trevor Pryce Leggett 1914-2000. Artículo online y disponible en The World of Judo magazine, URL: http://www.twoj. org/archives/2000/ autumn/aut00_news5.html (Consulta julio 2010). (Originalmente en: The World of Judo (1996). Edición no 24).

International Judo Federation (2010). World Kata Championships 2010 Budapest, Hungary, Competition Rules. Budapest: International Judo Federation.

International Judo Federation Data Commission (2010a). Kata Competition 2010. International Judo Federation.

Jomantas, N. (2009). Team USA to compete at first ever Kata World Championships this weekend (13 October 2009). Artículo online y disponible en USA Judo, URL: http://judo.teamusa.org/news/ article/28266 (Consulta julio 2010).

Jones, L. (2005). Competition, kata and the art of judo. Journal of Asian Martial Arts, 14(3), 72-85.

Kano, J. (s.f.). En Daigo, T. editor. (1964 y 2008). Kodokan Judo no kata (The kata of Kodokan judo). Tokyo: Kodokan Publications. Reimpreso para el 2008 Kodokan Summer Kata Course. Tokyo: Kodokan Judo Institute.

Kano, J. (1899). Kodokan Judo kogi (Conferencias de Judo Kodokan). Kokushi, 1(4). (En Japonés).

Kano, J. (1915). Kodokan Judo gaisetsu (Boceto de Judo Kodokan). Judo. 2. (En Japonés).

Kano, J. (1927). Kano Jigoro judoka, 12 (Kano Jigoro el judoka, parte 12). Sakko. 5(12). (En Japonés).

Kano, J. (1986). Kodokan judo. Tokyo: Kodansha International.

Kano, Y. (2007). W elcome Address. Souvenir Program of the 1st Kodokan Judo Kata International Tournament 2007. pp. 26-27.

Kawamura, T. \& Daigo, T. (Eds.) (2000). Kodokan new Japanese-English dictionary of judo. Tokyo: Kodokan Institute.

Klens-Bigman, D. (s.f.). Creativity, bound flow and the concept of shu-ha-ri in kata. Artículo en línea disponible en FightingArts.com, URL: http:// fightingarts. com/reading/article. php?id=173 (Consulta julio 2010).

Kodokan (1957-1999). Qualification for dan promotion. Información online y disponible en la web del Kodokan, URL: http://www.kodokan. org/e_basic/ shoudan.html (Consulta junio 2010).

Kodokan (1961). Judo. Osaka: Nunoi Shobo Co.

Kotani, S., Osawa, Y. \& Hirose, Y. (1968). Kata of Kodokan judo. Kobe: Koyano Bussan Kaisha Ltd. 
Kotani, S. \& Otaki, T. (1971). En Daigo, T. editor. (2008). Kodokan Goshinjutsu por [Saishin judo no kata]. Reimpreso para el 2008 Kodokan Kata Kaki Koshukai (Curso de Verano de Katas Kodokan). Tokyo: Kodokan Judo Institute.

Mori, 0. (2008). An introduction to Go no kata. Tokyo: publicación privada basada en Kuhara, Y. (1976). Judo Mizu Nagare. Tokyo: Shudokan Kuhara Dojo. (en japonés).

Murata, N. (2007). W hat is kata? The congratulatory address to the 1st Kodokan Judo Kata International Tournament. Souvenir Program of the 1st Kodokan Judo Kata International Tournament 2007, 26-27.

Murata, N. \& Todo, Y. (2007). A study on evaluation of judo kata performance. Bulletin of the Association for the Scientific Studies on Judo, Kodokan. Report XI. (En japonés, resumen en inglés).

Murata, N.\& Todo, Y. (2009). A study on evaluation of judo kata performance II Koshiki no Kata. Bulletin of the Association for the Scientific Studies on Judo, Kodokan. Report XII. (En japonés, resumen en inglés).

Nogaki, P. (2009). Correspondencia personal con Paul Nogaki sobre los ideales de la kata.

O da, J. (1929). Judo taikan. Tokyo: Shoshikan. (En japonés).

Oltremari, A. (s.f.). Kata, sport e gare (Kata, deporte y competición). Artículo en línea disponible en la web de freeBudo, URL:http://www. freebudo.com/ articoli/judo\%20tradizionale/articoli\%20judo/1\%20kata\% 20sport\%20 e\%20gare/1\%20kata\%20sport\%20e\%20gare.htm (En italiano) (Consulta julio 2010).

Otaki, T. \& Draeger, D. (1983). Judo formal techniques. Tokyo: Charles E. Tuttle Company.

Pelletier, G. (s.f.). Kata. Artículo online y disponible en la web de Guy Pelletier \& André Andermatt, URL: http://pagesperso-orange.fr/pelletier. andermatt/5kata0.html (en francés) (Consulta julio 2010).

Sheedy, J. (2010). Judo kata competitions: A review of practice strategies for the Goshin Jitsu [sic]. Artículo online y disponible en Judo Information website, URL: http://judoinfo.com/new/techniques/formsof-judo-kata/671practice-strategies-for-goshin-jitsu (Consulta julio 2010).

Sidney, J. (2003). The warrior's path: W isdom from contemporary martial arts masters. Boston: Shambhala Publications.

Takamura, Y. (1986). Teaching and shu-ha-ri. Artículo online y disponible en en la web de la Takamura-ha Shindo Yoshin Kai, URL: http://www. shinyokai. com/essays_teachingShuhari.htm (Consulta julio 2010).

Ukichi, S. (s.f.) Matsumoto, T. and Davidson, P. traductores. (s.f.), en Daigo, T. editor. (2008). Eternal kendo. Reimpreso para el 2008 Kodokan Kata Kaki Koshukai (Curso de Verano de Katas Kodokan). Tokyo: Kodokan Judo Institute. 